\title{
Quality parameters of wild white trevally (Pseudocaranx dentex) natural spawn kept in captivity
}

\author{
Natacha Nogueira $^{\mathrm{a}, *}$, Marilyn Ferreira ${ }^{\mathrm{a}}$, Nereida Cordeiro ${ }^{\mathrm{b}}$, Paula Canada ${ }^{\mathrm{a}}$ \\ a Centro de Maricultura da Calheta, Direcção Regional de Pescas, Vila da Calheta, 9370-133 Calheta, Portugal \\ ${ }^{\mathrm{b}}$ Faculty of Exact Sciences and Engineering, University of Madeira, 9000-390 Funchal, Madeira, Portugal
}

\section{A R T I C L E I N F O}

\section{Keywords:}

Pseudocaranx dentex

Natural spawning

Egg quality

Lipids and fatty acid profile

Viability parameters

\begin{abstract}
A B S T R A C T
The white trevally (Pseudocaranx dentex Bloch \& Schneider, 1801) is a widely distributed carangid, commercially exploited in both the Pacific and eastern Atlantic. Most published works refer to the use of hormonal induction with gonadotropin-releasing hormone for egg supply with generally low fecundities and of poor quality. This study aimed to contribute for a better knowledge on the husbandry conditions that allow for natural spawning of white trevally in captivity. Wild white trevallies were kept in captivity for four years until natural spawning occurred. Nine breeders were kept in a $10 \mathrm{~m}^{3}$ concrete tank at a density of $5 \mathrm{Kg} / \mathrm{m}^{3}$, under natural photoperiod and natural water temperature fluctuations. Spawning started when water temperature reached $19^{\circ} \mathrm{C}$ and lasted for two months. Viability rates varied between 35 and $79 \%$ and the average number of spawned eggs per female was of $280 \times 10^{3}$. Egg total lipids $(16 \% \mathrm{DW})$ and fatty acid profile (expressed as a \% total detected) remained constant throughout the spawning season and were not correlated with any egg viability parameters. Neutral lipids, a major energy source in marine fish eggs and larvae, accounted for $50 \%$ of lipid classes, followed by glycolipids and phospholipids. Within lipid fractions, the neutral lipids presented high amounts of monounsaturated fatty acids followed by equally high contents of polyunsaturated fatty acids. Docohexaenoic acid (DHA) was mainly found in the phospholipids fraction, though it was present in high amounts in all lipid classes. However, no significant correlations were observed between the DHA content and the egg viability parameters. The percentage of arachidonic acid (Ara) $(<0.1 \%$ ) was low, possibly due to a deficiency of this fatty acid in the broodstock diet. Polyunsaturated fatty acids content, mostly represented by DHA and eicosapentaenoic acid (EPA), was positively correlated with hatching rate. Correlations observed between chemical composition of the eggs and viability parameters highlight the fact that egg quality is deeply influenced not only by environmental factors but also by broodstock management techniques.
\end{abstract}

\section{Introduction}

There is a worldwide increasing demand for more sustainable and profitable aquatic products. Thus, the development of new and competitive products is of paramount importance if aquaculture is to boost production in Europe. Carangids have received attention in various parts of the world during the past 30 years as potential new species for aquaculture diversification. The white trevally (Pseudocaranx dentex Bloch \& Schneider, 1801) is a widely distributed carangid, commercially exploited in both the Pacific and eastern Atlantic (Masuda and Tsukamoto, 2000; Scandol and Rowling, 2007; Smith-Vaniz et al., 2015). In Japan, there is a long history of white trevally off-shore cultivation and sea-ranching (FAO, 2006-2017; Harada, 1970; Harada et al., 1984; Kuwada et al., 2008) and induced spawning performance of white trevally kept in captivity fed different artificial diets has been previously described (Table 1). In Europe, white trevally culture is still in an embryonic status (Andrade et al., 2012; Roo et al., 2012) and a major constraint is the lack of a regular egg supply and most importantly good quality eggs. Roo et al. (2012) referred to the use of hormonal induction with gonadotropin-releasing hormone (GnRh) for egg supply, resulting in low fecundities and low hatching rates. Nevertheless, the collection of fertilized eggs obtained from natural spawning is a routine technique in aquaculture which minimizes the handling stress of broodstock fish (Bromage et al., 1994) and until now very few cases of white trevally spontaneous spawning under captivity have been reported (Andrade et al., 2012; Murai et al., 1987).

Good quality eggs have been generally defined as those exhibiting low mortalities at fertilization, hatching and first feeding (Bromage et al., 1992). More recently, Bobe and Labbe (2010) defined fish egg quality as the ability of the egg to be fertilized and subsequently

\footnotetext{
* Corresponding author.

E-mail address: natachacnogueira@gmail.com (N. Nogueira).
} 


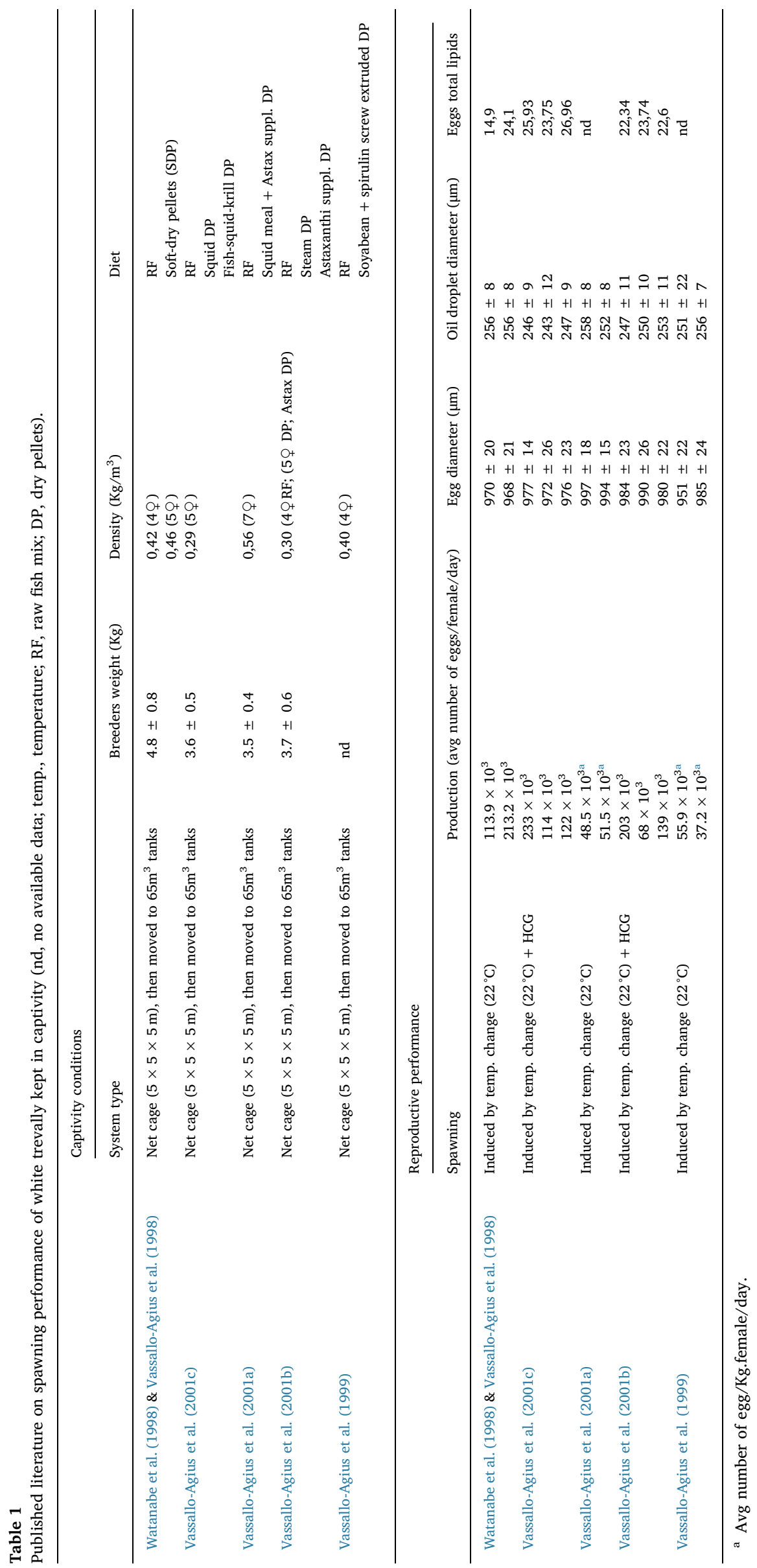


develop into a normal embryo, while Çoban et al. (2011) considered the potential of an egg to hatch into a viable larva. Despite the different definitions, it is accepted that numerous factors affect egg quality in fish, such as environmental parameters (Lahnsteiner and Kletzl, 2012); broodstock diet (Almansa et al., 1999; Bromage et al., 1992; Furuita et al., 2002; Izquierdo et al., 2001; Zakeri et al., 2009); husbandry practices (Bromage, 1995) and the domestication level of the species (Bobe and Labbe, 2010; Migaud et al., 2013). Several physical, biological and biochemical parameters are used as indicators of broodstock reproductive performance and egg quality (Bromage, 1995). Besides fertilization and hatching rates, parameters such as buoyancy, egg size, distribution of lipid droplets and blastomere morphology have been cited as reliable indicators of egg quality (Aristizabal et al., 2009; Kohn and Symonds, 2012; Mansour et al., 2008). In addition, biochemical parameters, such as eggs fatty-acid composition, in particular n-3 HUFA concentrations (Almansa et al., 1999; Bruce et al., 1999; FernándezPalacios et al., 1995; Furuita et al., 2002; Navas et al., 1997) and n-3/n6, DHA/ArA, EPA/ArA ratios (Bell and Sargent, 2003; Pickova et al., 1997; Tveiten et al., 2004) were suggested to affect egg quality and larval survival in several species. The fatty-acid composition of white trevally eggs obtained by induced spawning after hormonal treatments and/or water temperature manipulation has been characterized and compared between broodstocks fed different formulated diets in Japan (Vassallo-Agius et al., 1998, 1999, 2001a, 2001b, 2001c; Watanabe et al., 1998).

If white trevally is to be seen as a candidate for the European aquaculture diversification, the ability to control eggs production and quality is a major requirement for the sustainable cultivation of this species. A full control of sexual maturation and spawning under captivity is a primary requirement for broodstock management and good farming practice and it is often achieved through environmental manipulation (Bromage, 1995). The knowledge of environmental conditions allowing for spontaneous spawning under captivity and the characterization of egg quality under such conditions may be a useful reference to establish a protocol for environmental manipulation towards the control of reproduction of Eastern Atlantic white trevally. As mentioned above, egg quality is deeply influenced not only by environmental factors and broodstock management techniques, but also by the aquaculture system used and the domestication level of the species or the population and these factors may act in a species-dependent manner. The present study was not a nutritional trial but rather sought to associate and characterize white trevally natural spawning performance under standard operating procedures in a marine hatchery using morphological and chemical analyses as indicators of egg quality, further increasing knowledge on white trevally culture techniques.

\section{Material and methods}

\subsection{Broodstock and rearing system}

White trevally individuals (Pseudocaranx dentex) were captured off the south coast of Madeira Archipelago (Portugal) and transported to the Mariculture Center of Calheta, a Governmental Marine Hatchery Center. Fish were kept in a $10 \mathrm{~m}^{3}$ concrete tank under natural photoperiod with constant water renewal. Salinity was $36 \%$ and temperature ranged from 18 to $24^{\circ} \mathrm{C}$ year-round. Fish were fed near satiation once a day, six days a week with a moist pelleted diet- Lansy Breed Maturation, INVE (Table 2), supplemented twice a week with a mixture of low commercial value diet, mainly chub mackerel (Scomber japonicus), european squid (Loligo vulgaris) and blue mussel (Mytilus edulis) at a ratio of 2:2:1. All the individuals were weighted every year before the spawning season. After four years in captivity, natural spawning
Table 2

Proximate composition of Lansy Breed Maturation moist pelleted diet.

\begin{tabular}{ll}
\hline Moisture & $22 \%$ \\
Crude protein & $40 \%$ \\
Crude ash & $12 \%$ \\
Crude lipids & $15 \%$ \\
Crude fiber & $1 \%$ \\
Phosphorus & $1.3 \%$ \\
Vit. A & $11.000 \mathrm{IU} / \mathrm{Kg}$ \\
Vit. D3 & $2.100 \mathrm{IU} / \mathrm{Kg}$ \\
Vit. E & $2.100 \mathrm{IU} / \mathrm{Kg}$ \\
Vit. C & $4.100 \mathrm{IU} / \mathrm{Kg}$ \\
DHA/EPA & 2 \\
$\Sigma \omega 3 H U F A$ & $36 \mathrm{mg} / \mathrm{g} \mathrm{dwt}$ \\
\end{tabular}

occurred and the broodstock was kept at a density of $5.073 \mathrm{~kg} / \mathrm{m}^{3}$, with a total of nine breeders (4Males:5Females).

\subsection{Reproductive performance}

Broodstock reproductive performance was evaluated by the total number of spawning events, total number of eggs, proportion of viable (buoyant) and unviable (sinking) eggs, fertilization rate and hatching rate. During the spawning season, an egg collector tank (100 L), with an $800 \mu \mathrm{m}$ mesh size bag, was placed by the side opening of the broodstock tank. Floating eggs were collected by overflow and stocked in the collector tank with flowing water and air supply. The collector tank was monitored every morning, at $9 \mathrm{am}$. Whenever spawning had occurred, the eggs were collected, placed in a $5 \mathrm{~L}$ bucket with saltwater for total number and viability estimation. In each spawning event, the total number of spawned eggs and viability rates were estimated according to Fernández-Palacios et al. (1995). Ten samples of $5 \mathrm{~mL}$ were observed under the stereoscope. In each $5 \mathrm{~mL}$ sample, two fractions could be distinguished: a fraction of viable buoyant eggs and a second fraction of sinking eggs (non-viable). Viable fertilized eggs were described as the percentage of morphologically normal buoyant eggs at the morula stage and described as transparent, perfectly spherical with clear, symmetrical early cleavages (Kjørsvik et al., 1990).

The total numbers of viable eggs (TVE), unviable eggs (TUE), spawned eggs (TE), egg viability rate (EVR, \%) and fertilization rate (EFR, \%) were calculated as:

TVE $=($ bucket volume $\times$ Mean total viable egg $) \times 5 \mathrm{~mL}$

TUE $=($ bucket volume $\times$ Mean total unviable egg $) \times 5 \mathrm{~mL}$

$\mathrm{TE}=\mathrm{TVE}+\mathrm{TUE}$

$\operatorname{EVR}(\%)=(\mathrm{TVE} \times 100) / \mathrm{TE}$

EFR $(\%)=($ number of normal and fertilized eggs

\section{/Total viable buoyant fraction) $\times 100$}

The hatching rate (HR, \%) was evaluated once a week, in triplicate. One hundred viable (buoyant) eggs were disinfected with $1.5 \mathrm{~mL} / \mathrm{L}$ of formaldehyde for $5 \mathrm{~min}$ and stocked in three $2 \mathrm{~L}$ aquariums filled with filtered water and gentle aeration. Dead eggs were counted and removed daily until hatching (approximately $48 \mathrm{~h}$ ). Hatching rate (HR, $\%)$ was calculated as:

HR $(\%)=$ number of hatched larvae

$\times 100 /$ number of viable (buoyant) eggs 


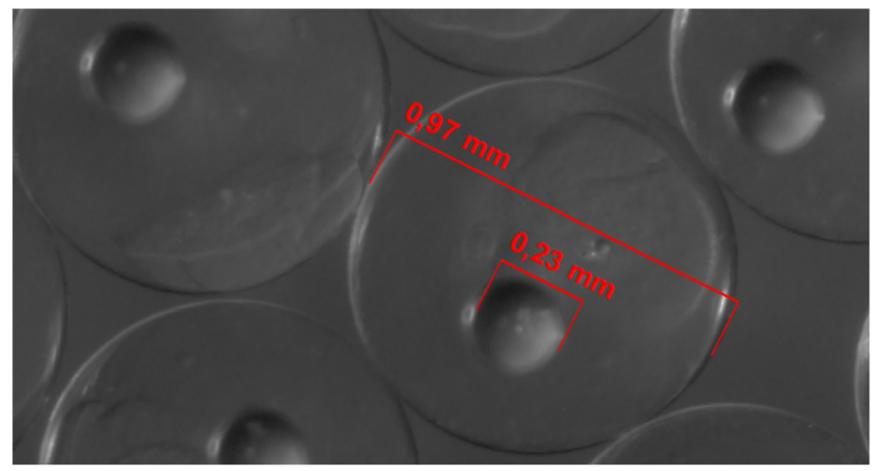

Fig. 1. Measurement of white trevally egg and oil droplet diameters.

\subsection{Egg measurements}

Weekly samples of thirty eggs were collected, placed under the stereoscope (Zeiss DV4) and photographed. Egg and oil droplet diameters were measured, using Axio Vision L.E. 4.3 (Carl Zeiss Micro Imaging $\mathrm{GmbH}$ ), with a precision of $0.1 \mathrm{~mm}$ (Fig. 1).

\subsection{Biochemical analyses}

In order to perform biochemical analysis, approximately $1 \mathrm{~g}$ (WW) of eggs was collected once a week, using a $75-\mu \mathrm{m}$ mesh filter. The egg pool was snap frozen in liquid nitrogen and stored at $-80^{\circ} \mathrm{C}$ until further analysis. All the samples were freeze-dried until residual water percentage was below $3 \%$. The lipid content was determined according to Bligh and Dyer (1959) and described in Fernandes et al. (2016). From each freeze-dried egg pool, $100 \mathrm{mg}$ were weighted and added $3 \mathrm{~mL}$ of a solution containing methanol/chloroform/BHT (2:1:0.01\%) and $400 \mu \mathrm{L}$ of saturated solution of potassium chloride. Two $\mathrm{mL}$ of chloroform and $2 \mathrm{~mL}$ of distilled water were then added and the mixture was placed under agitation for $20 \mathrm{~min}$. The organic phase was collected and the solvent removed using a rotary evaporator at $40^{\circ} \mathrm{C}$. The lipid content was determined gravimetrically and the results expressed in percentage of dry weight (DW).

Total lipid extracts were analysed for their fatty acid composition as fatty acid methyl esters (FAMEs) as previously described by Lepage and Roy (1986), modified by Cohen et al. (1988). Briefly, the fatty acids were converted to FAMEs by adding a mixture of ethyl acetate-methanol $(1: 19 \mathrm{v} / \mathrm{v})$ to total lipid aliquots that were after submitted at $80^{\circ} \mathrm{C}$ for $1 \mathrm{~h}$. FAMEs were analysed by gas chromatography (Agilent HP 6890) equipped with a mass selective detector (Agilent 5973) and a fused silica capillary column SupelcowaxTM $10(30 \mathrm{~m} \times 0.25 \mathrm{~mm}$ inner diameter, $0.25 \mu \mathrm{m}$ film thickness) from Supelco. The chromatographic conditions were: initial temperature, $40^{\circ} \mathrm{C}$ for $5 \mathrm{~min}$; temperature gradient, $2{ }^{\circ} \mathrm{C} \mathrm{min}{ }^{-1}$; final temperature, $250{ }^{\circ} \mathrm{C}$ for $5 \mathrm{~min}$; injector temperature, $260^{\circ} \mathrm{C}$; transfer-line temperature, $260^{\circ} \mathrm{C}$; split ratio, $1: 100$. Helium was used as the carrier gas with a flow of $1.0 \mathrm{~mL} \mathrm{~min}^{-1}$. The FAMEs were identified through comparison of retention times and mass spectra obtained with two standard samples: "bacterial acid methyl esters CP mix" and "Supelco 37 component FAME mix" and spectra library Wiley-NIST. Heneicosanoic acid as an internal standard. The results were expressed in percentage of total FA detected, being the quantification made according to the response factor determined for each FA present in the standards, in comparison with the heneicosanoic acid (internal standard).
Five egg samples were used for determination of lipid classes (neutral lipids, glycolipids and phospholipids), according to Fernandes et al. (2016). Previously extracted lipids were solubilized in dichloromethane and fractionated in activated silica chromatography column (at $100^{\circ} \mathrm{C}$ ). The separation of lipid classes was made in the following elution sequence: $5 \mathrm{~mL}$ of dichloromethane (neutral lipids, $\mathrm{NL}$ ), $5 \mathrm{~mL}$ of acetone (glycolipids, GL) and, $10 \mathrm{~mL}$ of methanol (phospholipids, PL). All chemical analyses were carried out at least in duplicate.

\subsection{Data analyses}

The statistical analyses followed previously reported methods (Zar, 2010) and IBM SPSS Statistics 23 was the software used for all the statistical analysis performed. All data were tested for normality using a Kolmogorov-Smirnov (whenever $\mathrm{n}>30$ ) or Shapiro-Wilk (whenever $\mathrm{n}<30$ ) test, and for homogeneity of variance using a Levene's test. Biochemical data were log transformed to fit a normal distribution and all the percentage data were arcsine transformed prior to analyses. Means and back-transformed means are presented and the standard errors are those obtained from untransformed variables. The differences between weeks in egg quality parameters, total lipids, total fatty acids content, lipid classes (neutral lipids, phospholipids and glycolipids) and each class fatty acids content were tested by a one-way ANOVA, followed by Tukey's multiple comparison test. A Pearson's coefficient correlation was applied to establish the relationship between the egg morphological and performance parameters and biochemical composition. Significant levels were set at $P<0.05$.

\section{Results}

The natural spawning of wild white trevally kept in captivity lasted approximately two months (from May 5th to June 29th), when water temperatures ranged from $19.5^{\circ} \mathrm{C}$ to $21.9^{\circ} \mathrm{C}$ and photoperiod was of $16 \mathrm{~h}$ of light. There were 20 spawning events and the total number of eggs collected was estimated at 10.8 million (10 789780 ), of which $42.90 \%$ were unviable (sinking) eggs and $56.63 \%$ were viable (buoyant) eggs. The number of eggs collected at each event varied between 15.600 and 1.430 .400 with an average number of eggs per batch of 674.361 . The egg production, mean viability rate and fertilization rate are shown in Fig. 2. The total number of eggs and the viability rate were highly variable and there was no clear trend throughout the spawning season. Still, hatching rate variations correlated to water temperature (Table 3). Hatching rate was also correlated to total monounsaturated fatty acids (MUFA) and polyunsaturated fatty acids contents (Table 3). The fertilization rate was always higher than $95 \%$ (Fig. 2).

The white trevally eggs were spherical, non-adhesive and pelagic with a mean diameter of $0.969 \pm 0.027 \mathrm{~mm}$ and an oil globule of $0.279 \pm 0.017 \mathrm{~mm}$. The mean weekly egg diameter fluctuated without significant differences between weeks $(P>0.05)$. However, the average egg size declined towards the end of the season (Fig. 3) and was negatively correlated with water temperature (Table 3).

Lipid content was fairly constant throughout the spawning season with a mean value of $16.63 \pm 1.01 \%$ DW (Table 4). There were no correlations between the eggs lipid content and the number of viable eggs, the egg size or oil droplet size. Fatty acid (FA) profile was also fairly constant throughout the spawning season. Polyunsaturated fatty acids (PUFA) were the major component of total lipids, representing $>40 \%$ of total detected FA, followed by saturated fatty acids (SFA, 27-29\%) and monounsaturated fatty acids (MUFA, 

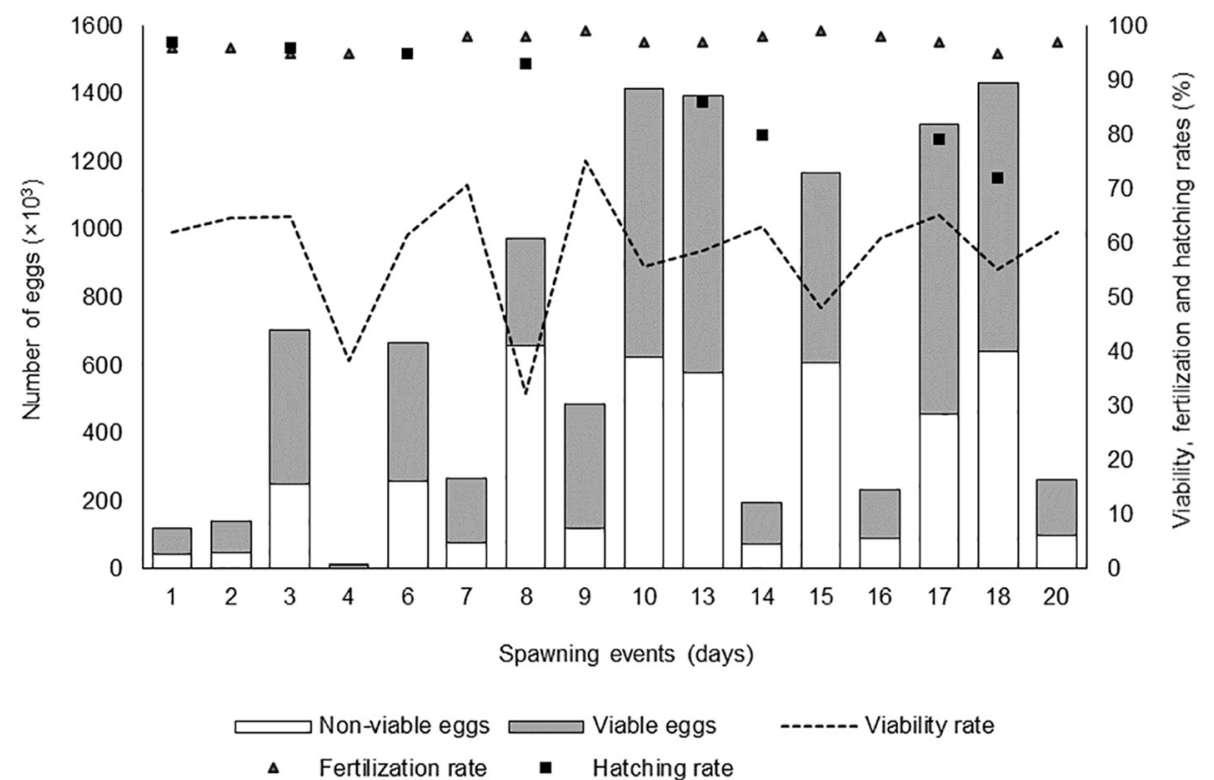

Fig. 2. Total number of spawned viable (buoyant) and unviable (sinking) eggs (left Y-axis), viability, fertilization, hatching rate (\%) (right Y-axis) throughout the reproductive season of white trevally.

Table 3

Correlations (Pearson's coefficients) for temperature, eggs morphological and performance parameters and biochemical composition (monounsaturated fatty acids MUFA, polyunsaturated fatty acids - PUFA, and docosahexaenoic acid - DHA - contents, proportion of total lipids). Pearson's coefficients (r) and corresponding significance level (P) and number of entries (n) are presented for each correlation. Statistical significance was set at $\mathrm{P}<0.05$.

\begin{tabular}{|c|c|c|c|c|c|c|c|}
\hline & Temperature & Viable eggs & Egg diam. & Hatching rate & MUFA & PUFA & DHA \\
\hline Viable eggs & $\mathrm{P}=0.044, \mathrm{r}=0.202(\mathrm{n}=210)$ & - & & & & & \\
\hline Egg diam. & $\mathrm{P}=0.040, \mathrm{r}=-0.197(\mathrm{n}=210)$ & ns & - & & & & \\
\hline MUFA & $\mathrm{P}=0.017, \mathrm{r}=0.941(\mathrm{n}=11)$ & ns & ns & $\mathrm{P}=0.040, \mathrm{r}=-0.894(\mathrm{n}=11)$ & - & & \\
\hline PUFA & Ns & ns & ns & $\mathrm{P}=0.032, \mathrm{r}=0.909(\mathrm{n}=11)$ & $P=0.030, r=-0.802(n=11)$ & - & \\
\hline DHA & $\mathrm{P}=0.042, \mathrm{r}=-0.892(\mathrm{n}=11)$ & ns & ns & Ns & $P=0.007, r=-0.887(n=11)$ & ns & - \\
\hline
\end{tabular}

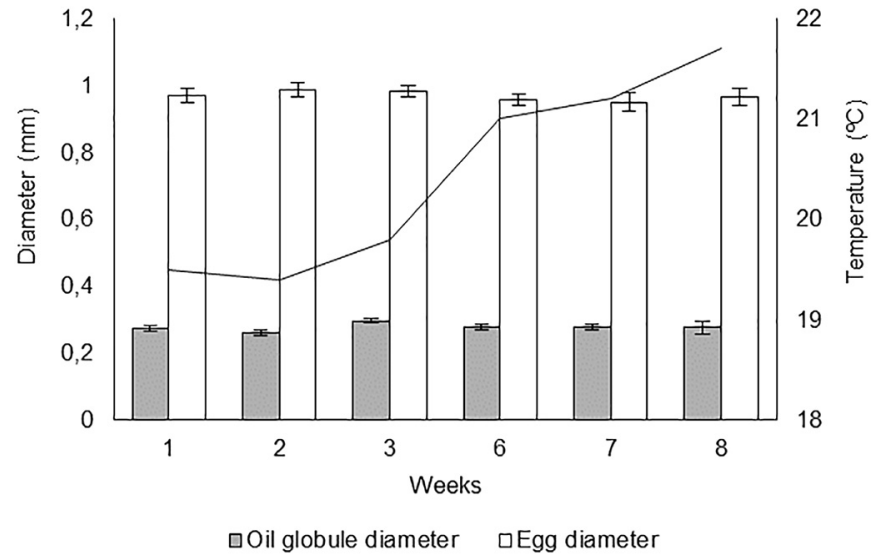

Fig. 3. Egg and oil globule diameter (left Y-axis) and temperature (right Y-axis) throughout the reproductive season of white trevally. Values are means \pm s.d. $(\mathrm{n}=30)$. No statistitical differences $(\mathrm{P}>0.05,1$ way-ANOVA) were found between weeks.
24-27\%) with no significant differences being detected between weeks (Fig. 4). Within each major fatty acid group, palmitic acid (16:0) accounted for $52.58 \%$ of SFA, oleic acid (18:1) accounted for $73.61 \%$ of

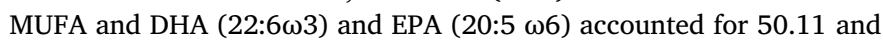
$13.32 \%$ of PUFA, respectively.

Lipids class composition in white trevally eggs was fairly constant throughout spawning season ( $P>0.05$ ) (Fig. 5). Neutral lipids (NL) fraction accounted for $>49 \%$ of total lipids during the entire spawning season, followed by glycolipids (GL) (23.5-26.5\%) and polar lipids (18.6-22.8\%). Again, no correlation was observed between lipid classes and other egg quality parameters. The fatty acid profile varied between lipids classes: whereas MUFA were mostly represented in the neutral and glycolipid fractions (Tables 5, 6), SFA were mostly abundant in PL (Table 7). GL fraction presented the lowest amount of PUFA, particularly EPA and DHA (Table 6), which were mostly abundant in the PL fraction (Table 7).

\section{Discussion}

In fish, egg quality is a key factor to the successful production of 
Table 4

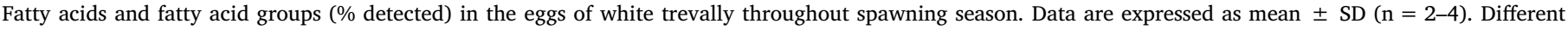
letters within the same row represent statistical differences $(\mathrm{P}<0.05)$.

\begin{tabular}{|c|c|c|c|c|c|c|c|c|}
\hline & \multicolumn{8}{|l|}{ Weeks } \\
\hline & 1 & 2 & 3 & 4 & 5 & 6 & 7 & 8 \\
\hline Total lipids (\%DW) & $16.37 \pm 1.45$ & $16.79 \pm 1.23$ & $16.87 \pm 1.29$ & $16.40 \pm 1.26$ & $17.63 \pm 0.98$ & $15.74 \pm 0.36$ & $17.57 \pm 0.27$ & $16.14 \pm 1.22$ \\
\hline \multicolumn{9}{|l|}{ Fatty acids (\%) } \\
\hline $\mathrm{C} 14: 0$ & $1.76 \pm 0.09^{\mathrm{a}}$ & $1.80 \pm 0.013^{\mathrm{a}}$ & $1.77 \pm 0.02^{\mathrm{a}}$ & $1.88 \pm 0.38^{\mathrm{ab}}$ & $1.81 \pm 0.03^{\mathrm{a}}$ & $1.77 \pm 0.02^{\mathrm{a}}$ & $1.89 \pm 0.01^{\mathrm{ab}}$ & $2.04 \pm 0.06^{\mathrm{b}}$ \\
\hline $\mathrm{C} 16: 0$ & $14.65 \pm 0.49$ & $14.38 \pm 0.10$ & $14.48 \pm 0.59$ & $15.10 \pm 0.22$ & $13.99 \pm 0.36$ & $14.50 \pm 0.02$ & $14.49 \pm 0.20$ & $14.48 \pm 0.25$ \\
\hline C18:0 & $3.72 \pm 0.16$ & $3.74 \pm 0.10$ & $3.70 \pm 0.28$ & $3.92 \pm 0.04$ & $3.60 \pm 0.11$ & $3.87 \pm 0.05$ & $3.62 \pm 0.10$ & $4.00 \pm 0.25$ \\
\hline Others & $7.41 \pm 1.23$ & $7.14 \pm 0.07$ & $8.39 \pm 1.33$ & $6.99 \pm 0.15$ & $7.30 \pm 0.07$ & $8.64 \pm 0.01$ & $7.51 \pm 0.19$ & $6.47 \pm 0.47$ \\
\hline Total - SFA & $27.54 \pm 1.62$ & $27.06 \pm 0.06$ & $28.34 \pm 1.46$ & $27.90 \pm 0.07$ & $26.69 \pm 0.43$ & $28.79 \pm 0.05$ & $27.50 \pm 0.10$ & $27.00 \pm 0.35$ \\
\hline C16:1n-9 & $5.53 \pm 0.38$ & $5.33 \pm 0.04$ & $5.40 \pm 0.10$ & $5.60 \pm 0.05$ & $5.27 \pm 0.04$ & $5.25 \pm 0.01$ & $5.51 \pm 0.07$ & $5.60 \pm 0.12$ \\
\hline C18: 1n-9 & $17.57 \pm 1.36^{\mathrm{a}}$ & $18.69 \pm 0.15^{\mathrm{a}}$ & $17.83 \pm 0.88^{\mathrm{a}}$ & $18.90 \pm 0.14^{\mathrm{a}}$ & $18.16 \pm 0.20^{\mathrm{a}}$ & $18.61 \pm 0.10^{\mathrm{a}}$ & $19.73 \pm 0.51^{\mathrm{b}}$ & $19.50 \pm 0.24^{b}$ \\
\hline C20:1 & $0.83 \pm 0.08^{\mathrm{ab}}$ & $0.89 \pm 0.01^{\mathrm{a}}$ & $0.98 \pm 0.05^{\mathrm{bcd}}$ & $0.92 \pm 0.01^{\mathrm{b}}$ & $1.03 \pm 0.01^{\mathrm{d}}$ & $0.97 \pm 0.01^{\mathrm{c}}$ & $1.11 \pm 0.04^{\mathrm{e}}$ & $1.13 \pm 0.03^{\mathrm{e}}$ \\
\hline Others & $0.133 \pm 0.02$ & $0.10 \pm 0.00$ & $0.31 \pm 0.22$ & $0.10 \pm 0.00$ & $0.13 \pm 0.00$ & $0.10 \pm 0.00$ & $0.13 \pm 0.00$ & $0.41 \pm 0.29$ \\
\hline Total - MUFA & $24.06 \pm 1.64$ & $24.98 \pm 0.20$ & $24.53 \pm 0.79$ & $25.51 \pm 0.19$ & $24.60 \pm 0.24$ & $24.93 \pm 0.13$ & $26.49 \pm 0.63$ & $26.64 \pm 0.54$ \\
\hline C18:2n6 & $5.58 \pm 1.89^{\mathrm{a}}$ & $6.20 \pm 0.08^{\mathrm{ac}}$ & $6.47 \pm 0.08^{\mathrm{ac}}$ & $5.88 \pm 0.03^{\mathrm{ab}}$ & $5.98 \pm 0.07^{\mathrm{abc}}$ & $6.15 \pm 0.01^{\mathrm{ac}}$ & $6.76 \pm 0.15^{\mathrm{ad}}$ & $6.27 \pm 0.27^{\mathrm{ac}}$ \\
\hline C18:3n3 & $0.69 \pm 0.00^{\mathrm{a}}$ & $0.71 \pm 0.00^{\mathrm{b}}$ & $0.77 \pm 0.02^{c}$ & $0.69 \pm 0.00^{\mathrm{a}}$ & $0.71 \pm 0.01^{\mathrm{b}}$ & $0.70 \pm 0.01^{\mathrm{ab}}$ & $0.74 \pm 0.01^{c}$ & $0.73 \pm 0.02^{\mathrm{bc}}$ \\
\hline $\mathrm{C} 18: 4 \mathrm{n} 3$ & $2.85 \pm 0.02^{\mathrm{a}}$ & $2.91 \pm 0.03^{\mathrm{b}}$ & $3.00 \pm 0.09^{\mathrm{bd}}$ & $2.75 \pm 0.04^{\mathrm{c}}$ & $2.98 \pm 0.02^{\mathrm{d}}$ & $2.86 \pm 0.01^{\mathrm{a}}$ & $3.02 \pm 0.02^{\mathrm{d}}$ & $2.68 \pm 0.12^{\mathrm{c}}$ \\
\hline C20:4n 6 & $<0.1$ & $<0.1$ & $<0.1$ & $<0.1$ & $<0.1$ & $<0.1$ & $<0.1$ & $<0.1$ \\
\hline $\mathrm{C} 20: 4 \mathrm{n} 3$ & $0.69 \pm 0.01^{\mathrm{a}}$ & $0.67 \pm 0.01^{\mathrm{ab}}$ & $0.70 \pm 0.02^{\mathrm{ac}}$ & $0.66 \pm 0.01^{\mathrm{b}}$ & $0.75 \pm 0.04^{c}$ & $0.67 \pm 0.01^{\mathrm{ab}}$ & $0.68 \pm 0.03^{\mathrm{abc}}$ & $0.68 \pm 0.02^{\mathrm{ab}}$ \\
\hline C20:5n 3 - EPA & $6.16 \pm 0.18^{c}$ & $5.36 \pm 0.02^{\mathrm{ab}}$ & $5.54 \pm 0.38^{\mathrm{b}}$ & $5.42 \pm 0.04^{\mathrm{b}}$ & $5.37 \pm 0.11^{\mathrm{b}}$ & $5.42 \pm 0.01^{\mathrm{b}}$ & $5.08 \pm 0.12^{\mathrm{a}}$ & $5.43 \pm 0.26^{\mathrm{b}}$ \\
\hline C22:5n6 & $0.47 \pm 0.12$ & $0.54 \pm 0.01$ & $0.52 \pm 0.08$ & $0.51 \pm 0.01$ & $0.56 \pm 0.02$ & $0.55 \pm 0.02$ & $0.47 \pm 0.01$ & $0.50 \pm 0.03$ \\
\hline C22:5n3 & $0.94 \pm 1.08$ & $1.67 \pm 0.01$ & $0.91 \pm 0.05$ & $1.64 \pm 0.02$ & $1.78 \pm 0.08$ & $0.96 \pm 0.01$ & $1.71 \pm 0.04$ & $1.70 \pm 0.04$ \\
\hline C22:6n3- DHA & $22.39 \pm 1.52$ & $20.70 \pm 0.05$ & $20.89 \pm 1.07$ & $20.21 \pm 1.70$ & $21.59 \pm 0.51$ & $20.91 \pm 0.03$ & $19.58 \pm 0.40$ & $19.59 \pm 0.63$ \\
\hline Others & $2.75 \pm 0.19^{\mathrm{ab}}$ & $2.94 \pm 0.01^{b c}$ & $2.69 \pm 0.08^{\mathrm{a}}$ & $2.99 \pm 0.04^{c}$ & $2.93 \pm 0.06^{\mathrm{bc}}$ & $2.86 \pm 0.03^{b c}$ & $2.91 \pm 0.05^{c}$ & $2.58 \pm 0.13^{\mathrm{a}}$ \\
\hline Total - PUFA & $42.65 \pm 3.21$ & $41.89 \pm 0.01$ & $41.67 \pm 2.13$ & $40.94 \pm 0.30$ & $42.83 \pm 0.44$ & $40.34 \pm 0.02$ & $41.14 \pm 0.84$ & $40.35 \pm 0.54$ \\
\hline$\Sigma \mathrm{n} 3$ & $35.45 \pm 3.03$ & $33.81 \pm 0.05$ & $33.30 \pm 2.57$ & $33.14 \pm 0.28$ & $34.85 \pm 0.48$ & $32.27 \pm 0.01$ & $32.39 \pm 0.67$ & $32.27 \pm 0.71$ \\
\hline$\Sigma \mathrm{n} 6$ & $6.64 \pm 0.34^{\mathrm{b}}$ & $7.47 \pm 0.07^{\mathrm{e}}$ & $7.72 \pm 0.43^{\text {ade }}$ & $7.12 \pm 0.01^{\mathrm{c}}$ & $7.27 \pm 0.05^{\mathrm{d}}$ & $7.42 \pm 0.04^{\mathrm{e}}$ & $8.02 \pm 0.17^{\mathrm{a}}$ & $7.37 \pm 0.33^{\text {cde }}$ \\
\hline$\Sigma \mathrm{n} 3 / \Sigma \mathrm{n} 6$ & $5.33 \pm 0.19^{\mathrm{b}}$ & $4.52 \pm 0.05^{\mathrm{e}}$ & $4.34 \pm 0.57^{\text {cde }}$ & $4.66 \pm 0.04^{c}$ & $4.79 \pm 0.10^{c}$ & $4.35 \pm 0.02^{\mathrm{d}}$ & $4.04 \pm 0.01^{\mathrm{a}}$ & $4.39 \pm 0.28^{\mathrm{de}}$ \\
\hline DHA/EPA & $3.66 \pm 0.14^{\mathrm{b}}$ & $3.87 \pm 0.01^{\mathrm{c}}$ & $3.77 \pm 0.08^{\mathrm{ab}}$ & $3.73 \pm 0.01^{\mathrm{b}}$ & $4.02 \pm 0.18^{\mathrm{a}}$ & $3.86 \pm 0.01^{\mathrm{ac}}$ & $3.86 \pm 0.01^{\mathrm{ac}}$ & $3.61 \pm 0.11^{\mathrm{b}}$ \\
\hline
\end{tabular}

Limit of detection for all fatty acids: $0.1 \% ; \mathrm{n}=22$. SFA- saturated fatty acids; MUFA- monounsaturated fatty acids; PUFA- polyunsaturated fatty acids.

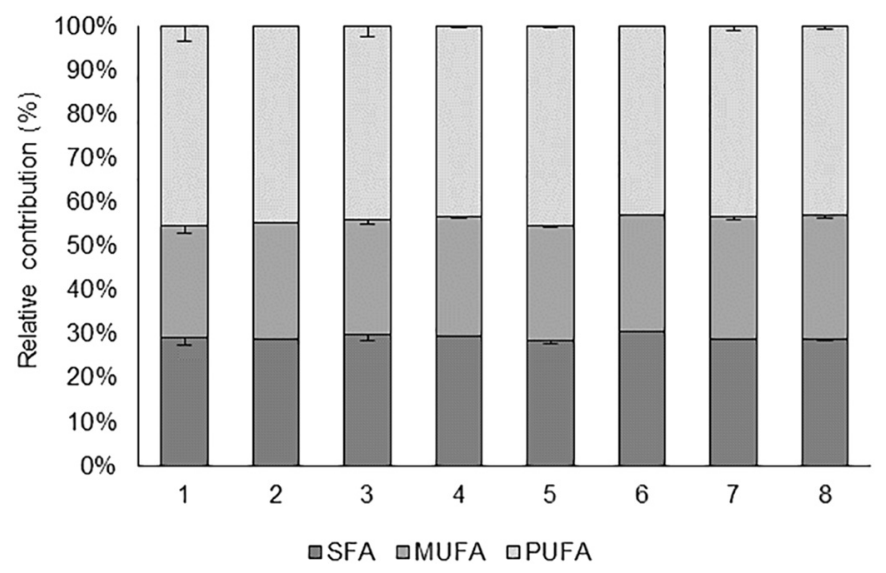

Fig. 4. Fatty acids groups, as a proportion of total lipids (\% detected) in white trevally eggs throughout spawning season. Values are means \pm s.d. $(n=10)$. No statistical differences ( $\mathrm{P}>0.05,1$ way-ANOVA) were found for each group between weeks.

viable offspring in marine teleosts (Jia et al., 2014). The present study aimed to describe the husbandry conditions and characterize the reproductive performance and egg quality of white trevally obtained by natural spawning in captivity.

Reference for the optimum temperatures for hatching and newlyhatched larval rearing of white trevally are between 20 and $22^{\circ} \mathrm{C}$ (Kawabe et al., 1991; Murai et al., 1992) which is within the range of Madeira archipelago natural seawater temperatures, $18-24^{\circ} \mathrm{C}$ (Gouveia et al., 2003). The present results show that after four years in captivity, wild white trevally fed commercial diets commonly used for broodstock

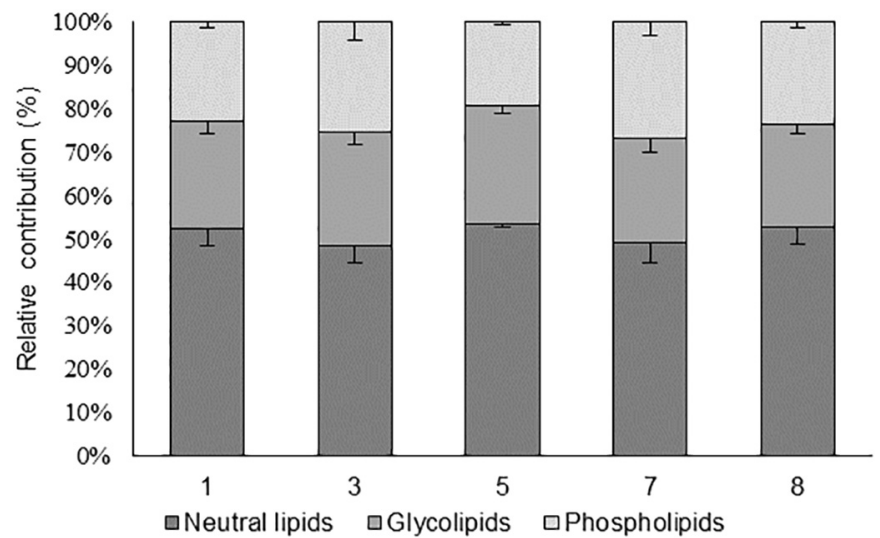

Fig. 5. Lipid class composition (\% detected) in white trevally eggs throughout spawning season. Values are means \pm s.d. $(n=10)$. No statististical differences ( $\mathrm{P}>$ 0.05, 1 way-ANOVA) were found for each class between weeks.

maintenance, supplemented with raw fish, were able to naturally spawn when water temperatures reached $19^{\circ}$. Spawning season was quite short ( 2 months) and stopped when the water temperature reached $22^{\circ}$, in agreement with what has been described in the wild for a Central North Atlantic population (Afonso et al., 2008).

Discrimination of "good" quality eggs by virtue of the eggs ability to float in seawater has long been used in many marine hatcheries (Carrillo et al., 1989; McEvoy, 1984). In the present study, the number of viable (buoyant) and unviable (sinking) eggs was variable throughout the spawning season and no correlation was detected with other egg quality parameters. The egg buoyancy has often been used as an indicator of egg quality in marine fish (Jia et al., 2014) and several 
Table 5

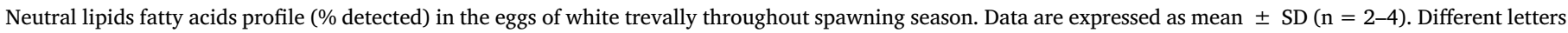
within the same row represent statistical differences $(P<0.05)$.

\begin{tabular}{|c|c|c|c|c|c|}
\hline & \multicolumn{5}{|l|}{ Weeks } \\
\hline & 1 & 3 & 5 & 7 & 8 \\
\hline \multicolumn{6}{|l|}{ Fatty acids (\%) } \\
\hline 14:0 & $2,03 \pm 0,15^{\mathrm{a}}$ & $1,88 \pm 0,02^{\mathrm{ab}}$ & $1,73 \pm 0,02^{\mathrm{b}}$ & $2,04 \pm 0,03^{\mathrm{a}}$ & $1,99 \pm 0,05^{\mathrm{ab}}$ \\
\hline $16: 0$ & $12,02 \pm 0,42^{\mathrm{bc}}$ & $11,51 \pm 0,06^{\mathrm{bc}}$ & $10,42 \pm 0,02^{\mathrm{c}}$ & $13,63 \pm 0,16^{\mathrm{a}}$ & $12,85 \pm 0,15^{\mathrm{a}}$ \\
\hline 18:0 & $1,53 \pm 0,21$ & $0,81 \pm 0,12$ & $1,02 \pm 0,15$ & $0,61 \pm 0,11$ & $2,58 \pm 1,63$ \\
\hline Outros & $0,48 \pm 0,10$ & $0,59 \pm 0,03$ & $0,59 \pm 0,00$ & $0,72 \pm 0,09$ & $0,54 \pm 0,11$ \\
\hline Total - SFA & $16,06 \pm 0,57^{\mathrm{abc}}$ & $14,78 \pm 0,24 b c$ & $13,75 \pm 0,19^{c}$ & $17,01 \pm 0,38^{\mathrm{ab}}$ & $17,96 \pm 1,58^{\mathrm{a}}$ \\
\hline $16: 1$ & $6,94 \pm 0,69$ & $6,32 \pm 0,12$ & $6,26 \pm 0,02$ & $7,07 \pm 0,12$ & $6,79 \pm 0,14$ \\
\hline $18: 1$ & $26,89 \pm 0,95^{\mathrm{a}}$ & $24,92 \pm 0,14^{\mathrm{ab}}$ & $23,65 \pm 0,17^{\mathrm{b}}$ & $27,22 \pm 1,07^{\mathrm{a}}$ & $25,13 \pm 0,88^{\mathrm{ab}}$ \\
\hline $20: 1$ & $1,17 \pm 0,52$ & $1,57 \pm 0,01$ & $1,67 \pm 0,00$ & $1,61 \pm 0,07$ & $1,13 \pm 0,52$ \\
\hline Outros & $11,69 \pm 0,82^{\mathrm{b}}$ & $10,87 \pm 0,08^{b}$ & $13,98 \pm 0,07^{\mathrm{a}}$ & $11,46 \pm 0,24^{\mathrm{b}}$ & $10,63 \pm 0,74^{\mathrm{b}}$ \\
\hline Total - MUFA & $46,69 \pm 1,53^{\mathrm{a}}$ & $43,67 \pm 0,07^{b}$ & $45,56 \pm 0,07^{\mathrm{ab}}$ & $47,35 \pm 0,64^{\mathrm{a}}$ & $43,68 \pm 0,63^{b}$ \\
\hline $18: 3 w 3$ & $1,22 \pm 0,42$ & $1,85 \pm 0,02$ & $1,54 \pm 0,01$ & $1,58 \pm 0,05$ & $1,81 \pm 0,07$ \\
\hline $18: 4 w 3$ & $4,61 \pm 0,23^{\mathrm{a}}$ & $3,89 \pm 0,02^{\mathrm{ab}}$ & $4,15 \pm 0,02^{\mathrm{ab}}$ & $3,54 \pm 0,23^{b}$ & $3,89 \pm 0,48^{\mathrm{ab}}$ \\
\hline $20: 4 w 3$ & $0,40 \pm 0,03^{\mathrm{b}}$ & $0,55 \pm 0,03^{\mathrm{a}}$ & $0,30 \pm 0,00^{\mathrm{bc}}$ & $0,59 \pm 0,09^{\mathrm{a}}$ & $0,20 \pm 0,05^{\mathrm{c}}$ \\
\hline 20:5w3 - EPA & $4,28 \pm 1,14$ & $4,26 \pm 0,05$ & $4,40 \pm 0,01$ & $3,23 \pm 0,13$ & $3,61 \pm 0,14$ \\
\hline $22: 5 w 3$ & $1,52 \pm 0,46$ & $1,52 \pm 0,07$ & $1,67 \pm 0,01$ & $1,15 \pm 0,05$ & $1,21 \pm 0,34$ \\
\hline $22: 6 w 3$ - DHA & $12,29 \pm 4,73$ & $11,34 \pm 0,26$ & $13,52 \pm 0,11$ & $8,04 \pm 0,08$ & $8,74 \pm 0,79$ \\
\hline Outros & $12,90 \pm 4,88$ & $18,14 \pm 0,05$ & $15,11 \pm 0,01$ & $17,52 \pm 0,25$ & $18,89 \pm 0,11$ \\
\hline Total - PUFA & $37,25 \pm 1,32^{\mathrm{c}}$ & $41,55 \pm 0,31^{\mathrm{a}}$ & $40,69 \pm 0,12^{\mathrm{ab}}$ & $35,65 \pm 0,26^{c}$ & $38,36 \pm 0,97^{\mathrm{bc}}$ \\
\hline$\Sigma w 3$ & $25,52 \pm 5,96$ & $24,46 \pm 0,22$ & $27,16 \pm 0,13$ & $19,38 \pm 0,12$ & $20,68 \pm 0,75$ \\
\hline ¿w6 & $0,11 \pm 0,13$ & $0,14 \pm 0,01$ & $0,25 \pm 0,00$ & $0,14 \pm 0,02$ & $0,07 \pm 0,09$ \\
\hline
\end{tabular}

Limit of detection for all fatty acids: $0.1 \% ; n=20$. SFA- saturated fatty acids; MUFA- monounsaturated fatty acids; PUFA- polyunsaturated fatty acids.

Table 6

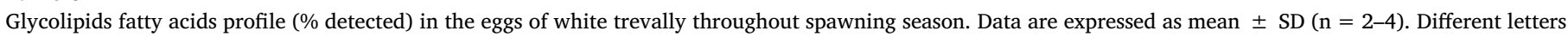
within the same row represent statistical differences $(\mathrm{P}<0.05)$.

\begin{tabular}{|c|c|c|c|c|c|}
\hline & \multicolumn{5}{|l|}{ Weeks } \\
\hline & 1 & 3 & 5 & 7 & 8 \\
\hline \multicolumn{6}{|l|}{ Fatty acids (\%) } \\
\hline $14: 0$ & $4,81 \pm 1,26$ & $4,08 \pm 0,14$ & $4,89 \pm 0,38$ & $3,63 \pm 0,07$ & $3,12 \pm 0,83$ \\
\hline $16: 0$ & $27,15 \pm 2,83$ & $28,17 \pm 0,09$ & $25,64 \pm 1,14$ & $26,82 \pm 0,41$ & $24,43 \pm 0,66$ \\
\hline $18: 0$ & $5,29 \pm 1,07$ & $5,70 \pm 0,07$ & $4,33 \pm 0,13$ & $5,51 \pm 0,09$ & $5,41 \pm 0,37$ \\
\hline Outros & $1,42 \pm 0,05$ & $1,63 \pm 0,07$ & $1,15 \pm 0,01$ & $1,24 \pm 0,03$ & $1,37 \pm 0,38$ \\
\hline Total - SFA & $38,67 \pm 3,00$ & $39,59 \pm 0,05$ & $36,01 \pm 1,40$ & $37,20 \pm 0,42$ & $34,33 \pm 1,49$ \\
\hline $16: 1$ & $7,10 \pm 2,44$ & $5,09 \pm 0,01$ & $7,99 \pm 0,33$ & $4,90 \pm 0,09$ & $4,68 \pm 1,75$ \\
\hline $18: 1$ & $25,97 \pm 3,67$ & $21,73 \pm 0,60$ & $26,11 \pm 0,01$ & $21,20 \pm 0,02$ & $20,42 \pm 3,51$ \\
\hline $20: 1$ & $1,52 \pm 0,59$ & $2,08 \pm 0,18$ & $0,95 \pm 0,12$ & $1,32 \pm 0,11$ & $1,87 \pm 0,57$ \\
\hline Outros & $7,80 \pm 7,51^{\mathrm{ab}}$ & $10,28 \pm 0,08^{\mathrm{ab}}$ & $2,01 \pm 0,04^{\mathrm{b}}$ & $12,01 \pm 0,11^{\mathrm{ab}}$ & $16,95 \pm 0,99^{\mathrm{a}}$ \\
\hline Total - MUFA & $42,39 \pm 2,23$ & $39,17 \pm 0,49$ & $37,06 \pm 0,25$ & $39,44 \pm 0,06$ & $43,91 \pm 4,84$ \\
\hline $18: 3 w 3$ & $0,00 \pm 0,00^{\mathrm{b}}$ & $0,00 \pm 0,00^{\mathrm{b}}$ & $0,49 \pm 0,03^{\mathrm{a}}$ & $0,00 \pm 0,00^{\mathrm{b}}$ & $0,00 \pm 0,00 b$ \\
\hline $18: 4 w 3$ & $3,14 \pm 3,15^{\mathrm{ab}}$ & $5,77 \pm 0,01^{\mathrm{ab}}$ & $1,06 \pm 0,11^{\mathrm{b}}$ & $5,48 \pm 0,00^{\mathrm{ab}}$ & $7,22 \pm 0,79^{a}$ \\
\hline $20: 4 w 3$ & $1,40 \pm 1,07$ & $1,58 \pm 0,08$ & $0,60 \pm 0,04$ & $1,17 \pm 0,13$ & $1,22 \pm 0,11$ \\
\hline 20:5w3 - EPA & $3,48 \pm 1,25$ & $1,33 \pm 0,01$ & $2,79 \pm 0,19$ & $1,48 \pm 0,02$ & $2,79 \pm 2,07$ \\
\hline $22: 5 w 3$ & $1,48 \pm 0,49$ & $3,42 \pm 0,31$ & $1,05 \pm 0,13$ & $2,87 \pm 0,08$ & $2,57 \pm 2,97$ \\
\hline $22: 6 w 3$ - DHA & $5,15 \pm 3,78^{\mathrm{ab}}$ & $1,15 \pm 0,11^{\mathrm{b}}$ & $10,24 \pm 1,19^{\mathrm{a}}$ & $5,73 \pm 0,18^{\mathrm{ab}}$ & $1,35 \pm 0,49^{\mathrm{b}}$ \\
\hline Outros & $5,29 \pm 0,09^{c}$ & $8,00 \pm 0,03^{b}$ & $10,70 \pm 0,03^{\mathrm{a}}$ & $6,63 \pm 0,01^{\mathrm{bc}}$ & $6,61 \pm 1,04^{\mathrm{bc}}$ \\
\hline Total - PUFA & $18,94 \pm 1,28$ & $21,24 \pm 0,54$ & $26,93 \pm 1,65$ & $23,37 \pm 0,36$ & $21,75 \pm 6,30$ \\
\hline इw3 & $13,99 \pm 1,63$ & $13,24 \pm 0,51$ & $16,63 \pm 1,61$ & $16,73 \pm 0,37$ & $15,84 \pm 4,48$ \\
\hline$\Sigma w 6$ & n.d & n.d. & n.d. & n.d. & n.d. \\
\hline
\end{tabular}

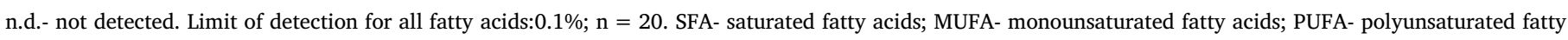
acids.

authors have reported that the ratio of buoyant eggs to total eggs spawned correlates positively with egg hatchability (Furuita et al., 2007; Unuma et al., 2005). In this study, no correlation was observed between hatching rate and egg buoyancy. Though the average number of produced eggs per female was comparable to the previously published literature (Table 1), the viability rates were lower. Hatching rate of white trevally decreased towards the end of the spawning season, in agreement to what has been described for many marine fish species in the wild (Chambers, 1977; Mihelakakis et al., 2001).

Both the hatching rate and egg size were negatively correlated with water temperature, decreasing towards the end of the spawning season. Explanations for these observed correlations include: a possible decline in sperm quality (Cabrita et al., 2011); differences between female's contribution in egg production during the spawning period (Fujita and 
Table 7

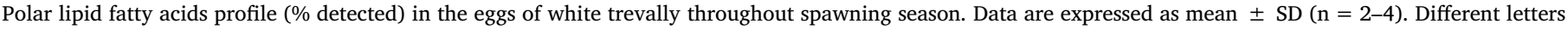
within the same row represent statistical differences $(P<0.05)$.

\begin{tabular}{|c|c|c|c|c|c|}
\hline & Weeks & & & & \\
\hline & 1 & 3 & 5 & 7 & 8 \\
\hline \multicolumn{6}{|l|}{ Fatty acids (\%) } \\
\hline 14:0 & $0,87 \pm 1,00^{\mathrm{b}}$ & $1,16 \pm 0,07^{\mathrm{b}}$ & $4,89 \pm 0,38^{\mathrm{a}}$ & $1,14 \pm 0,12^{\mathrm{b}}$ & $1,16 \pm 0,58^{b}$ \\
\hline $16: 0$ & $28,92 \pm 1,59$ & $30,90 \pm 0,56$ & $25,64 \pm 1,14$ & $29,39 \pm 1,15$ & $29,80 \pm 5,01$ \\
\hline $18: 0$ & $12,88 \pm 3,94^{\mathrm{a}}$ & $0,00 \pm 0,00^{\mathrm{b}}$ & $4,33 \pm 0,13^{b}$ & $16,13 \pm 0,10^{\mathrm{a}}$ & $19,33 \pm 1,99^{\mathrm{a}}$ \\
\hline Outros & $0,43 \pm 0,50$ & $0,94 \pm 0,10$ & $1,15 \pm 0,01$ & $0,87 \pm 0,02$ & $0,49 \pm 0,58$ \\
\hline Total - SFA & $43,10 \pm 1,73^{\mathrm{abc}}$ & $33,01 \pm 0,39^{c}$ & $36,01 \pm 1,40^{b c}$ & $47,54 \pm 1,20^{\mathrm{ab}}$ & $50,79 \pm 6,96^{\mathrm{a}}$ \\
\hline $16: 1$ & $1,58 \pm 1,83^{\mathrm{b}}$ & $2,40 \pm 0,04^{\mathrm{b}}$ & $7,99 \pm 0,33^{\mathrm{a}}$ & $2,84 \pm 0,13^{\mathrm{b}}$ & $1,86 \pm 0,56^{\mathrm{b}}$ \\
\hline $18: 1$ & $13,99 \pm 0,61^{\mathrm{b}}$ & $29,49 \pm 0,06^{\mathrm{a}}$ & $26,11 \pm 0,01^{\mathrm{a}}$ & $16,79 \pm 0,06^{\mathrm{b}}$ & $16,60 \pm 2,98^{b}$ \\
\hline $20: 1$ & $0,00 \pm 0,00^{c}$ & $0,61 \pm 0,03^{\mathrm{b}}$ & $0,95 \pm 0,12^{\mathrm{a}}$ & $0,00 \pm 0,00^{c}$ & $0,00 \pm 0,00^{c}$ \\
\hline Outros & $2,29 \pm 2,28$ & $1,12 \pm 0,13$ & $2,01 \pm 0,04$ & $2,35 \pm 0,12$ & $2,55 \pm 1,05$ \\
\hline Total - MUFA & $17,86 \pm 1,07^{\mathrm{b}}$ & $33,63 \pm 0,15^{\mathrm{a}}$ & $37,06 \pm 0,25^{\mathrm{a}}$ & $21,98 \pm 0,19^{b}$ & $21,00 \pm 4,56^{\mathrm{b}}$ \\
\hline $18: 3 w 3$ & $0,00 \pm 0,00$ & $0,00 \pm 0,00$ & $0,00 \pm 0,00$ & $0,00 \pm 0,00$ & $0,00 \pm 0,00$ \\
\hline $18: 4 w 3$ & $1,68 \pm 0,53$ & $1,13 \pm 0,18$ & $1,06 \pm 0,11$ & $1,37 \pm 0,25$ & $0,75 \pm 0,86$ \\
\hline $20: 4 w 3$ & $0,00 \pm 0,00$ & $0,00 \pm 0,00$ & $0,00 \pm 0,00$ & $0,00 \pm 0,00$ & $0,00 \pm 0,00$ \\
\hline 20:5w3 - EPA & $15,13 \pm 9,95$ & $4,45 \pm 0,10$ & $2,79 \pm 0,19$ & $3,81 \pm 0,32$ & $3,08 \pm 2,05$ \\
\hline $22: 5 w 3$ & $0,67 \pm 0,79$ & $0,88 \pm 0,06$ & $1,05 \pm 0,13$ & $0,90 \pm 0,04$ & $0,55 \pm 0,64$ \\
\hline 22:6w3 - DHA & $17,98 \pm 12,43$ & $19,03 \pm 0,04$ & $10,24 \pm 1,19$ & $16,25 \pm 1,04$ & $16,50 \pm 7,31$ \\
\hline Outros & $3,58 \pm 0,47 c$ & $7,88 \pm 0,05 b$ & $10,70 \pm 0,03 a$ & $8,15 \pm 0,23 b$ & $7,34 \pm 0,65 b$ \\
\hline Total - PUFA & $39,04 \pm 2,71$ & $33,37 \pm 0,24$ & $26,93 \pm 1,65$ & $30,49 \pm 1,38$ & $28,21 \pm 11,38$ \\
\hline ¿w3 & $35,47 \pm 3,07$ & $25,49 \pm 0,29$ & $16,63 \pm 1,61$ & $22,34 \pm 1,15$ & $20,87 \pm 10,85$ \\
\hline इw6 & n.d. & n.d. & n.d. & n.d. & n.d. \\
\hline
\end{tabular}

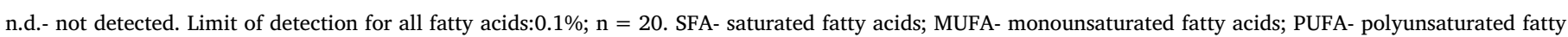
acids.

Yogata, 1984); depletion of female energy reserves (Chambers, 1977); or a general reduction in egg quality as the spawning period continues (Brooks et al., 1997).

The biochemical composition of fish eggs is related to their quality, as egg composition must satisfy embryonic nutritional needs for development and growth (Lubzens et al., 2010; Tocher, 2003). Typically, total lipid content (TL) of marine fish eggs ranges from 15 to $35 \%$ DW (Dayal et al., 2003; Samaee et al., 2009; Sargent et al., 1995) and may increase, decrease or remain constant during the spawning season (Aegerter and Jalabert, 2004; Faulk and Holt, 2008; Fuiman and Ojanguren, 2011). In this study, though white trevally eggs TL content remained fairly constant throughout the spawning season (approx. 16\% DW), it was lower than what was previously reported (Vassallo-Agius et al., 1998, 2001b, 2001c). In addition to being related with broodstock dietary lipids, eggs TL has also been suggested to be positively correlated with broodstock weight (Quiñones-Arreola et al., 2015). Since the broodstock individuals used in Vassallo-Agius et al. (1998, 2001b, 2001c) trials had lower mean bodyweight than the ones used in our study and the fact that diets used by those authors had higher lipid content (Table 1), the present results highlight the instrumental importance of dietary lipids as affecting egg lipid and fatty acid content (Sargent et al., 1995).

Moreover, similarly to what was observed in sole, sea bass, turbot (Devauchelle et al., 1982) and common dentex (Samaee et al., 2009), egg TL content was not correlated to egg size, oil droplet size or viability rate. On the contrary, TL was reported as being correlated with egg size in almaco jack (Quiñones-Arreola et al., 2015) and with viability in roach and bream (Wiegand, 1996). These contradictory results highlight the so far unclear relationship between egg total lipid content and egg size and viability in marine fish species, making it difficult to conclude whether these parameters directly affect egg quality of white trevally.

Within lipids composition, neutral lipids (NL) generally considered as a major energy reserve in marine fish eggs and larvae (Blaxter, 1988; Tocher, 2003), accounted for $50 \%$ of lipid classes. The high NL content found in this study reflects the relative increased size of the oil droplet as compared to egg size (approximately 30\%), since oil droplet of marine pelagic fish eggs typically consists mostly of neutral lipids (Tocher, 2003; Wiegand, 1996). Moreover, glycolipids, which are important cellular membrane components for anchoring/sorting of membrane structures and signaling transduction (Simons and Ikonen, 1997), are also present as droplets in the yolk of teleost eggs (Sarasquete et al., 2002), contributing as well for the size of the oil droplet.

Fatty acids (FA) composition of white trevally eggs fitted the general marine fish FA profile (Sargent et al., 1999, 2002) and agreed with previous results for the same species (Vassallo-Agius et al., 1998, 2001b, 2001c). FA composition was fairly constant throughout the spawning season, without any relevant differences in the major fatty acid groups. Significant differences were found in palmitic acid (16:0) and oleic acid (18:1), which were the most represented fatty acids in the SFA and MUFA fractions, respectively. However, these FA contents were not correlated with any egg viability parameters.

Hatching rate was negatively correlated to both temperature and MUFA content. Accordingly, PUFA content, mostly represented by DHA (22:6n-3) and EPA (20:5n-3), was positively correlated with hatching rate, which agrees to what has been described for most marine fish species (Fernández-Palacios et al., 2011). The fact that hatching rate was not directly correlated with DHA content, the most abundant fatty acid in PUFAs fraction, highlights the importance of other essential unsaturated fatty acids in determining egg quality parameters. Still, DHA content was negatively correlated with water temperature, which is in agreement with Mejri et al. (2014) that reported a positive correlation between the degree of unsaturation of fatty acids and membrane fluidity. Mejri et al. (2014) suggested that a decrease in DHA content towards the end of the spawning season could be an adaptive mechanism to reduce membrane fluidity with the increase of temperature.

Arachidonic acid (ArA) content also correlated with egg quality in many other species (Bell et al., 1997; Mazorra et al., 2003; Salze et al., 2005; Tocher and Sargent, 1984), was always very low throughout the spawning season (Table 4) when compared to what has been described for most marine fish species eggs (Fernández-Palacios et al., 2011). Since egg lipid composition is directly affected by the parental diet (Bell 
et al., 1997; Sargent et al., 1995), low detected levels of ArA in this study suggest that the proportion of this FA in the feed supplied to the brood stock could be unfitting for the quality of white trevally eggs.

As a consequence of low ArA levels and high EPA and DHA content, the $n-3 / n-6$ HUFA ratio was higher than what is commonly found in most marine fish (2.9) (Henderson and Tocher, 1987). Despite these results, no significant relationship was detected between the sum of n-3 HUFA or $n-3 / n-6$ ratio and number of buoyant viable eggs or any other egg quality measurements.

Within lipid fractions, the neutral lipids (NL) presented high amounts of MUFA followed by equally high contents of PUFA, which has been suggested to be a good indicator of offspring quality in fresh water species (Mejri et al., 2014). MUFA were also the most abundant FA in the glycolipid fraction (GL). However, in the GL fraction, SFA were more abundant than PUFA, mainly due to an increased amount of 16:0. In fact, GL displayed the lowest amounts of EPA and DHA. DHA was mainly found in the phospholipids (PL) fraction, confirming the important role of PUFA as structural and functional components in the cellular membrane (Tocher, 2003).

Despite no significant correlation was found between DHA percentage in all lipid classes and the viability parameters, the high relative abundance of DHA in all classes confirms the need for this FA in the developing embryos. DHA is particularly important for the membrane development in neural tissue, which comprises a relatively high proportion of tissues in rapidly developing embryos and larvae (Seaborn et al., 2009).

\section{Conclusion}

Egg quality control is crucial in species that have been "recently" introduced in the fish culturing systems, in order to characterize the husbandry practices that allow for the production of high quality larvae and juveniles, which is the key for the sustainability of a commercial hatchery.

In the present study, husbandry conditions differed from what has been previously published for this species. White trevallies were kept in smaller tanks at much higher densities, under natural photoperiod and natural water temperature fluctuations. Though spawning season was short, the number of spawned eggs per female was higher when compared with induced spawning of trevallies fed artificial diets. Correlations observed between the chemical composition of the eggs and viability parameters highlight the importance of broodstock nutrition and environmental parameters, such as water temperature on egg quality parameters. The present results suggest a combined and possibly synergistic effect of several parameters affecting egg quality, bringing up the need for a more appropriate experimental approach to investigate these biological interactions.

\section{Acknowledgments}

This study was partially supported by the Oceanic Observatory of Madeira (M1420-01-0145-FEDER-000001-Observatório Oceânico da Madeira-OOM).

\section{References}

Aegerter, S., Jalabert, B., 2004. Effects of post-ovulatory oocyte ageing and temperature on egg quality and on the occurrence of triploid fry in rainbow trout, Oncorhynchus mykiss. Aquaculture 231, 59-71.

Afonso, P., Fontes, J., Morato, T., Holland, K.N., Santos, R.S., 2008. Reproduction and spawning habitat of white trevally, Pseudocaranx dentex, in the Azores, central north Atlantic. Sci. Mar. 72, 373-381.

Almansa, E., Pérez, M.J., Cejas, J.R., Badia, P., Villamandos, J.E., Lorenzo, A., 1999. Influence of broodstock gilthead seabream (Sparus aurata L.) dietary fatty acids on egg quality and egg fatty acid composition throughout the spawning season. Aquaculture 170, 326-336.

Andrade, C.A., Nogueira, N., Silva, P., Dinis, M.T., Narciso, L., 2012. Mesocosm hatcheries using semi-intensive methodologies and species diversification in aquaculture. J.
Agric. Sci. Technol. B 2, 428-437.

Aristizabal, E., Suarez, J., Vega, A., Bargas, R., 2009. Egg and larval quality assessment in the Argentinean red porgy (Pagrus pagrus). Aquaculture 287, 329-334.

Bell, J.G., Sargent, J.R., 2003. Arachidonic acid in aquaculture feed: current status and future opportunities. Aquaculture 218, 491-499.

Bell, J.G., Tocher, D.R., Farndale, B.M., Cox, D.I., McKinney, R.W., Sargent, J.R., 1997. The effect of dietary lipid on polyunsaturated fatty acid metabolism in Atlantic salmon (Salmo salar) undergoing parr-smolt transformation. Lipids 32, 515-525.

Blaxter, J.H.S., 1988. Pattern and variety in development. In: Hoar, W.S., Randall, D.J. (Eds.), Fish Physiology. The Physiology of Developing Fish: Eggs and Larvae, vol. 11 A. Academic Press, New York, USA, pp. 1-58.

Bligh, E.G., Dyer, W.J., 1959. A rapid method of total lipid extraction and purification. Can. J. Biochem. Physiol. 37, 911-917.

Bobe, J., Labbe, C., 2010. Egg and sperm quality in fish. Gen. Comp. Endocrinol. 165, 535-548.

Bromage, N., 1995. Broodstock management and seed quality - general considerations. In: Bromage, N.R., Roberts, R.J. (Eds.), Broodstock Management and Egg and Larval Quality. Blackwell Science Ltd, Oxford, UK, pp. 1-24.

Bromage, N., Jones, J., Randall, C., Thrush, M., Springate, J., Duston, J., Barker, G., 1992. Broodstock management, fecundity, egg quality and the timing of egg production in the rainbow trout (Oncorhynchus mykiss). Aquaculture 100, 141-166.

Bromage, N., Bruce, M., Basavaraja, N., Rana, K., Shields, R., Young, C., Dye, J., Smith, P., Gillespie, M., Gamble, J., 1994. Egg quality determinants in finfish the role of overripening with special reference to the timing of stripping in the Atlantic halibut Hippoglossus hippoglossus. J. World Aquacult. Soc. 25, 13-21.

Brooks, S., Tyler, C.R., Sumpter, J.P., 1997. Egg quality in fish: what makes a good egg? Rev. Fish Biol. Fish. 7, 387-416.

Bruce, M., Oyen, F., Bell, G., Asturiano, J.F., Farndale, B., Carrillo, M., Zanuy, S., Ramos, J., Bromage, N., 1999. Development of broodstock diets for the European Sea Bass (Dicentrarchus labrax) with special emphasis on the importance of n-3 and n-6 highly unsaturated fatty acid to reproductive performance. Aquaculture 177, 85-97.

Cabrita, E., Ma, S., Diogo, P., Martínez-Páramo, S., Sarasquete, C., Dinis, M.T., 2011. The influence of certain aminoacids and vitamins on post-thaw fish sperm motility, viability and DNA fragmentation. Anim. Reprod. Sci. 125, 189-195.

Carrillo, M., Bromage, N., Zanuy, S., Serrano, R., Prat, F., 1989. The effect of modifications in photoperiod on spawning time, ovarian development and egg quality in the sea bass (Dicentrarchus labrax L.). Aquaculture 81, 351-365.

Chambers, R.C., 1977. Environmental influences on egg and propagule sizes in marine fishes. In: Chambers, R.C., Trippel, E.A. (Eds.), Early Life History and Recruitment in Fish Populations. Chapman \& Hall, London, UK, pp. 63-102.

Coban, D., Okan Kamacı, H., Suzer, C., Yıldırım, S., Gökhan, A., Yıldırım Korkut, A., Saka, Ş., Fırat, K., 2011. Effect of some morphometric characteristics on egg quality in common dentex, Dentex dentex (Linneaus, 1758). Turk. J. Fish. Aquat. Sci. 11 $425-431$.

Cohen, Z., Vonshak, A., Richmond, A., 1988. Effect of environmental conditions on fatty acid composition of the red alga Porphyridium cruentum: correlation to growth rate. J. Phycol. 24, 328-332.

Dayal, J.S., Ahamad Ali, S., Thirunavukkarasu, A.R., Kailasam, M., Subburaj, R., 2003. Nutrient and amino acid profiles of egg and larvae of Asian seabass, Lates calcarifer (Bloch). Fish Physiol. Biochem. 29, 141-147.

Devauchelle, N., Brichon, G., Lamour, F., Stephan, G., 1982. Biochemical composition of ovules and fecund eggs of sea bass (Dicentrarchus labrax), sole (Solea vulgaris), and turbot (Scophthalmus maximus). In: Richter, C.J.J., Goos, H.J.T. (Eds.), Proceedings of the International Symposium on Reproductive Physiology of Fish. Center for Agricultural Publishing and Documentation, Wageningen, the Netherlands, pp. $155-157$.

FAO, 2006-2017. Natural aquaculture sector overview. Japan. In: Makino, M. (Ed.), National Aquaculture Sector Overview Fact Sheets. FAO Fisheries and Aquaculture Department, Rome.

Faulk, C.K., Holt, G.J., 2008. Biochemical composition and quality of captive-spawned cobia Rachycentron canadum eggs. Aquaculture 279, 70-76.

Fernandes, T., Fernandes, I., Andrade, C.A.P., Cordeiro, N., 2016. Marine microalgae growth and carbon partitioning as a function of nutrient availability. Bioresour. Technol. 214, 541-547.

Fernández-Palacios, H., Izquierdo, M.S., Robaina, L., Valencia, A., Salhi, M., Vergara, J.M., 1995. Effect of n-3 HUFA level in broodstock diets on egg quality of gilthead sea bream (Sparus aurata L.). Aquaculture 132, 325-337.

Fernández-Palacios, H., Norberg, B., Izquierdo, M.S., Hamre, H., 2011. Effects of broodstock diet on eggs and larvae. In: Holt, G. (Ed.), Larval Fish Nutrition. WilleyBlackwell and Sons, Inc., West Sussex, pp. 151-181.

Fuiman, L.A., Ojanguren, A.F., 2011. Fatty acid content of eggs determines antipredator performance of fish larvae. J. Exp. Mar. Biol. Ecol. 407, 155-165.

Fujita, S., Yogata, T., 1984. Induction of ovarian maturation, embryonic development and larvae and juveniles of the amberjack, Seriola aureovittata. Japan. J. Ichthyol. 30, 426-434.

Furuita, H., Tanaka, M., Yamamoto, T., Suzuki, N., Takeuchi, T., 2002. Effects of high levels of n-3 HUFA in broodstock diet on egg quality and egg fatty acid composition of Japanese flounder, Paralichthys olivaceus. Aquaculture 210, 323-333.

Furuita, H., Hori, K., Suzuki, Sugita, T., Yamamoto, T., 2007. Effect of n-3 and n-6 fatty acids in broodstock diet on reproduction and fatty acid composition of broodstock and eggs in the Japanese eel Anguilla japonica. Aquaculture 267, 55-61.

Gouveia, N.M., Andrade, C.A., Gouveia, L., 2003. Perspectives for tuna farming in Madeira archipelago. In: Bridges, C.R., García, A., Gordin, H. (Eds.), International symposium on Domestication of the bluefin tuna Thunnus thynnus. CIHEAM: Cahiers Options Mediterranéennes Zaragoza, pp. 81-82.

Harada, T., 1970. The present status of marine fish cultivation research in Japan. 
Helgoländer Meeresun. 20, 594-601.

Harada, T., Murata, O., Miyashita, S., 1984. Artificial hatching and rearing of striped jack, Caranx delicatissimus. Kinki Daigaku Suisan Kenkyujou Nenpo 2, 151-160.

Henderson, R.J., Tocher, D.R., 1987. The lipid composition and biochemistry of freshwater fish. Prog. Lipid Res. 26, 281-347.

Izquierdo, M.S., Fernandez-Palacios, H., Tacon, A.G.J., 2001. Effect of broodstock nutrition on reproductive performance of fish. Aquaculture 197, 25-42.

Jia, Y., Meng, Z., Liu, X., Lei, J., 2014. Biochemical composition and quality of turbot (Scophthalmus maximus) eggs throughout the reproductive season. Fish Physiol. Biochem. 40, 1093-1104.

Kawabe, K., Murai, M., Kato, K., Takashima, F., 1991. The effect of water temperature on egg development of striped jack Caranx delicatissimus. Suisan Zoshoku 39, 211-216.

Kjørsvik, E., Mangor-Jensen, A., Holmefjord, I., 1990. Egg quality in fishes. Adv. Mar. Biol. 26, 71-113.

Kohn, Y.Y., Symonds, J.E., 2012. Evaluation of egg quality parameters as predictors of hatching success and early larval survival in hapuku (Polyprion oxygeneios). Aquaculture 342, 42-47.

Kuwada, H., Masuda, R., Kobayashi, T., Kogane, T., Miyazaki, T., Imaizumi, K., Tsukamoto, K., 2008. Releasing Technique in Striped Jack Marine Ranching: PreRelease Acclimation and Presence of Decoys to Improve Recapture Rates, Stock Enhancement and Sea Ranching. Blackwell Publishing Ltd, pp. 106-116.

Lahnsteiner, F., Kletzl, M., 2012. The effect of water temperature on gamete maturation and gamete quality in the European grayling (Thymalus thymallus) based on experimental data and on data from wild populations. Fish Physiol. Biochem. 38, 455-467.

Lepage, G., Roy, C.C., 1986. Direct transesterification of all classes of lipids in a one-step reaction. J. Lipid Res. 27, 114-120.

Lubzens, E., Young, G., Bobe, J., Cerdà, J., 2010. Oogenesis in teleosts: how fish eggs are formed. Gen. Comp. Endocrinol. 165, 367-389.

Mansour, N., Lahnsteiner, F., McNiven, M.A., Richardson, G.F., 2008. Morphological characterization of Arctic char, Salvelinus alpinus, eggs subjected to rapid post-ovulatory aging at $7^{\circ} \mathrm{C}$. Aquaculture 279, 204-208.

Masuda, R., Tsukamoto, K., 2000. Onset of association behavior in striped jack, Pseudocaranx dentex, in relation to floating objects. Fish. Bull. 98, 864-869.

Mazorra, C., Bruce, M., Bell, J.G., Davie, A., Alorend, E., Jordan, N., Rees, J., Papanikos, N., Porter, M., Bromage, N., 2003. Dietary lipid enhancement of broodstock reproductive performance and egg and larval quality in Atlantic halibut (Hippoglossus hippoglossus). Aquaculture 227, 21-33.

McEvoy, L.A., 1984. Ovulatory rhytms and over-ripening of eggs in cultivated turbot, Scophtalmus maximum L. J. Fish Biol. 24, 437-448.

Mejri, S., Audet, C., Vandenberg, G.W., Parrish, C.C., Tremblay, R., 2014. Biochemical egg quality in a captive walleye (Sander vitreus) broodstock population relative to ovulation timing following hormonal treatment. Aquaculture 431, 99-106.

Migaud, H., Bell, G., Cabrita, E., McAndrew, B., Davie, A., Bobe, J., Herráez, M., Carrillo, M., 2013. Gamete quality and broodstock management in temperate fish. Rev. Aquac. 5, S194-S223.

Mihelakakis, A., Yoshimatsu, T., Tsolkas, C., 2001. Spawning in captivity and early life history of cultured red porgy, Pagrus pagrus. Aquaculture 199, 333-352.

Murai, M., Kato, K., Nakano, T., Takashima, F., 1987. Egg development and morphological changes of larvae of striped jack Caranx delicatissimus. Suisan Zoshoku 34, 217-226.

Murai, M., Kawabe, K., Takashima, F., 1992. Optimum salinity and temperature for hatching of striped jack egg. Aquac. Sci. 40, 261-268.

Navas, J.M., Bruce, M., Thrush, M., Farndale, B., Bromage, N., Zanuy, S., Carrillo, M., Bell, J.G., Ramos, J., 1997. The impact of seasonal alteration in the lipid composition of broodstock diets on egg quality in the European sea bass. J. Fish Biol. 51, 760-773.

Pickova, J., Dutta, P.C., Larsson, P.-O., Kiessling, A., 1997. Early embryonic cleavage pattern, hatching success and egg-lipid fatty acid composition: comparison between two cod (Gadus morhua) stocks. Can. J. Fish. Aquat. Sci. 54, 2410-2416.

Quiñones-Arreola, M.F., Arcos-Ortega, G.F., Gracia-López, V., Casillas-Hernández, R., Weirich, C., Morris, T., Díaz-Tenorio, M., Ibarra-Gámez, C., 2015. Reproductive broodstock performance and egg quality of wild caught and first-generation domesticated Seriola rivoliana reared under same culture conditions. Lat. Am. J. Aquat. Res. 43, 953-962.

Roo, J., Izquierdo, M.S., Mesa-Rodriguez, A., Guirao, R., Hernández-Cruz, C.M., Fernández-Palacios, H., 2012. Current status and recent advances in striped jack
Pseudocaranx dentex culture in Europe. Aqua 2012 (Prague, Czech Republic).

Salze, G., Tocher, D.R., Roy, W.J., Robertson, D.A., 2005. Egg quality determinants in cod (Gadus morhua L.): egg performance and lipids in eggs from farmed and wild broodstock. Aquac. Res. 36, 1488-1499.

Samaee, S., Estévez, A., Giménez, G., Lahnsteiner, F., 2009. Evaluation of quantitative importance of egg lipids and fatty acids during embryos and larvae development in marine pelagophil teleosts: with an emphasis on Dentex dentex. J. Exp. Zool. A Ecol. Genet. Physiol. 311A, 735-751.

Sarasquete, C., Cárdenas, S., González, C.M., Pascual, E., 2002. Oogenesis in the bluefin tuna, Thunnus thynnus L.: a histological and histochemical study. Histol. Histopathol. 17, 775-788.

Sargent, J.R., Bell, J.G., Bell, M.V., Henderson, R.J., Tocher, D.R., 1995. Requirement criteria for essential fatty acids. J. Appl. Ichthyol. 11, 183-198.

Sargent, J., McEvoy, L., Estevez, A., Bell, G., Bell, M., Henderson, J., Tocher, D., 1999. Lipid nutrition of marine fish during early development: current status and future directions. Aquaculture 179, 217-229.

Sargent, J.R., Tocher, D.R., Bell, J.G., 2002. The lipids. In: Halver, J.E., Hardy, R.W. (Eds.), Fish Nutrition, 3rd edition. Academic Press, San Diego, pp. 181-257.

Scandol, J., Rowling, K., 2007. Resource Assessments for Multi-Species Fisheries in NSW, Australia: Qualitative Status Determination Using Life History Characteristics, Empirical Indicators and Expert Review. Wild Fisheries Program; Systems Research Branch. Division of Science and Research; NSW Department of Primary Industries.

Seaborn, G.T., Smith, T.I.J., Denson, M.R., Walker, A.B., Berlinsky, D.L., 2009. Comparative fatty acid composition of eggs from wild and captive black sea bass, Centropristis striata L. Aquac. Res. 40, 656-668.

Simons, K., Ikonen, E., 1997. Functional rafts in cell membranes. Nature 387, 569-572.

Smith-Vaniz, W.F., Williams, J.T., Pina Amargos, F., Curtis, M., Brown, J., 2015. Pseudocaranx dentex, white trevally. In: I.U.f.C.o.N.a.N (Ed.), Resources. The IUCN Red List of Threatened Species (e.T190070A16643997).

Tocher, D.R., 2003. Metabolism and functions of lipids and fatty acids in teleost fish. Rev. Fish. Sci. 11, 107-184.

Tocher, D.R., Sargent, J.R., 1984. Analyses of lipids and fatty acids in ripe roes of some Northwest European marine fish. Lipids 19, 492-499.

Tveiten, H., Jobling, M., Andreassen, I., 2004. Influence of egg lipids and fatty acids on egg viability, and their utilization during embryonic development of spotted wolffish, Anarhichas minor. Aquac. Res. 35, 152-161.

Unuma, T., Kondo, S., Tanaka, H., Kagawa, H., Nomura, K., Ohta, H., 2005. Relationship between egg specific gravity and egg quality in the Japanese eel, Anguilla japonica. Aquaculture 246, 493-500.

Vassallo-Agius, R., Watanabe, T., Mushiake, K., Kawano, K., Satoh, S., 1998. Chemical components of eggs and yolksac larvae obtained from striped jack broodstock fed on a raw fish mix or dry pellets. Fish. Sci. 64, 759-765.

Vassallo-Agius, R., Mushiake, K., Imaizumi, H., Yamazaki, T., Watanabe, T., 1999. Spawning and quality of eggs of striped jack fed raw fish or dry pellets with $2 \%$ Spirulina. Suisan Zoshoku 47, 415-422.

Vassallo-Agius, R., Watanabe, T., Imaizumi, H., Yamazaki, T., Satoh, S., Kiron, V., 2001a. Effects of dry pellets containing astaxanthin and squid meal on the spawning performance of striped jack Pseudocaranx dentex. Fish. Sci. 67, 667-674.

Vassallo-Agius, R., Imaizumi, H., Watanbe, T., Yamazaki, T., Satoh, S., Kiron, V., 2001 b. The influence of astaxanthin supplemented dry pellets on spawning of striped jack. Fish. Sci. 67, 260-270.

Vassallo-Agius, R., Imaizumi, H., Watanabe, T., Yamazaki, T., Satoh, S., Kiron, V., 2001c. Effect of squid meal in dry pellets on the spawning performance of striped jack Pseudocaranx dentex. Fish. Sci. 67, 271-280.

Watanabe, T., Vassallo-Agius, R., Keiichi, M., Kawano, K., Kiron, V., Satoh, S., 1998. The first spawn-taking from striped jack broodstock fed soft-dry pellets. Fish. Sci. 64, 39-43.

Wiegand, M.D., 1996. Composition, accumulation and utilization of yolk lipids in teleost fish. Rev. Fish Biol. Fish. 6, 259-286.

Zakeri, M., Marammazi, J.G., Kochanian, P., Savari, A., Yavari, V., Haghi, M., 2009. Effects of protein and lipid concentrations in broodstock diets on growth, spawning performance and egg quality of yellowfin sea bream (Acanthopagrus latus). Aquaculture 295, 99-105.

Zar, J.H., 2010. Biostatistical Analysis. Pearson Prentice Hall. 